\title{
OpenMG: A New Multigrid Implementation in Python
}

\author{
Tom S. Bertalan ${ }^{\ddagger *}$, Akand W. Islam ${ }^{\ddagger}$, Roger B. Sidje ${ }^{\S}$, Eric Carlson
}

\begin{abstract}
In many large-scale computations, systems of equations arise in the form $A u=b$, where $A$ is a linear operation to be performed on the unknown data $u$, producing the known right-hand side, $b$, which represents some constraint of known or assumed behavior of the system being modeled. Since such systems can be very large, solving them directly can be too slow. In contrast, a multigrid solver solves partially at full resolution, and then solves directly only at low resolution. This creates a correction vector, which is then interpolated to full resolution, where it corrects the partial solution. This project aims to create an open-source multigrid solver called OpenMG, written only in Python. The existing PyAMG multigrid implementation is a highly versatile, configurable, blackbox solver, but is difficult to read and modify due to its $C$ core. Our proposed OpenMG is a pure Python experimentation environment for testing multigrid concepts, not a production solver. By making the code simple and modular, we make the algorithmic details clear. We thereby create an opportunity for education and experimentation with the partial solver (Jacobi, Gauss Seidel, SOR, etc.), the restriction mechanism, the prolongation mechanism, and the direct solver, or the use of GPGPUs, multiple CPUs, MPI, or grid computing. The resulting solver is tested on an implicit pressure reservoir simulation problem with satisfactory results.
\end{abstract}

Index Terms—-python, multigrid, numpy, partial differential equations

\section{Introduction to Multigrid}

Multigrid algorithms aim to accelerate the solution of large linear systems that typically arise from the discretization of partial differential equations. While small systems (hundreds of unknowns) can efficiently be solved with direct methods such as Gaussian elimination or iterative methods such as Gauss-Seidel, these methods do not scale well. In contrast, multigrid methods can theoretically solve a system in $O(N)$ CPU steps and memory usage [Brandt2].

The entire multigrid algorithm can be summarized in a few steps. The process below assumes that the user has first discretized the partial differential equation ("PDE") of interest, or otherwise expressed the problem as a matrix system of equations.

1) Setup hierarchies of operators and restriction matrices.

2) Find an approximation to the solution (pre-smooth the high-frequency error).

3) Find the fine residual.

4) Coarsen the residual, and produce the coarse right-hand side.

* Corresponding author: tom@tombertalan.com

* The University of Alabama, Department of Chemical and Biological Engineering

$\S$ The University of Alabama, Department of Mathematics

Copyright $(2012$ Tom S. Bertalan et al. This is an open-access article distributed under the terms of the Creative Commons Attribution License, which permits unrestricted use, distribution, and reproduction in any medium, provided the original author and source are credited.
5) Solve at the coarse level (via a direct solver or a recursive call to the multigrid algorithm).

6) Prolong the coarse solution, and produce the fine correction vector.

7) Return the corrected solution.

Because of the possibility for a recursive call, this is often called a multigrid "cycle".

The basic premise of multigrid is that a quick but sloppy solution can be corrected using information calculated at a coarser resolution. That is, an approximation is first made at the fine resolution, and the residual from this approximation is used as the right-hand side for a correction equation to be solved at a much coarser resolution, where computational costs are also much lower. This basic two-grid scheme can be extended by using a recursive call at the coarse level instead of a direct solver.

History

Multigrid techniques were first introduced in 1964 in the USSR by R. P. Fedorenko [Fedorenko1], [Fedorenko2], who recognized the significance of the interaction between the mesh resolution and the components of the error of an iterative solution (see section PreSmoothing), but who initially conceived of the multigrid algorithm simply as an occasional correction to a basic iterative solver. Rigorous analysis of the technique was furthered in the seventies by Achi Brandt, Wolfgang Hackbusch, and R. A. Nicolaides. Brandt [Brandt2] placed more emphasis on the coarse-grid representations, describing multigrid as a method by which to "intermix discretization and solution processes, thereby making both of them orders of magnitude more effective." He further recast the process in terms of local and global mode analysis (Fourier analysis) in 1994 [Brandt1]. In 1979 Nicolaides wrote a useful synthesis of the work of Fedorenko and Brandt up to that point, and also contrasted the older two-level coarse-grid correction strategy with true, $l$-level multigrid [Nicolaides]. Hackbrush wrote one of the foundational texts on multigrid [Hackbusch].

More information on the history of multigrid techniques can be found in several books [Trottenberg], [Hackbusch], [Wesseling] or lecture notes [Heckbert] on the topic.

Examples of simulation problem domains that have benefited from multigrid techniques include porous media transport [Douglas2], [Kameswaran], molecular dynamics [Dzwinel], [Zapata], [Boschitsch], fluid dynamics [Denev], [Douglas2], [Kameswaran], and neural network simulations (and neurotransmitter diffusion) [Bakshi].

Multigrid concepts are not limited to applications in simulation. Mipmapped textures for computer graphics and ultra-highresolution image viewing applications such as satellite imaging 
both rely on the concept of a hierarchy of grid resolutions. Here, intergrid transfer operators are used for the purpose of creating images at different resolutions than the original.

\section{Existing Python Implementations}

The current open-source Python multigrid implementation PyAMG (due to Nathan Bell [Bell]) is a very capable and speedy multigrid solver, with a core written in C. However, because of the extent of optimizations (and the inclusion of $\mathrm{C}$ code), it is not particularly readable.

Another interesting implementation is Yvan Notay's AGMG, which is available for Matlab and Fortran and includes parallel versions [Notay], [AGMG]. AGMG is available for free for academic use and by site-license for commercial use.

Our project, OpenMG, is not intended to be a production solver but instead a tool for education and experimentation. In this, it is largely inspired by the intentions behind Douglas, Deng, Haase, Liebmann, and Mckenzie's AMGlab [Douglas1], which is written for MATLAB. (AMGlab is freely available, although a license does not seem to be specified.) OpenMG is constructed in a modular fashion so each part can be understood by itself. Optimizations that might decrease readability have been avoided. Because of the modularity of the system, simplified components of the algorithm can be overridden with more optimized components in the future.

\section{Theoretical Algorithm}

\section{Discretization}

The need for any sort of linear algebraic solver arises when a system of partial differential equations is discretized on a finite grid of points. While this is not the work of the OpenMG solver itself (the arguments to the solver are already in discretized form), it is a necessary preliminary step.

A good illustration of discretization is that of the Poisson equation, $\nabla u=0$. Here, $\nabla$ is the Laplace operator, which signifies the sum of unmixed second partial derivatives.

$$
\nabla u=\frac{\partial^{2} u}{\partial x^{2}}+\frac{\partial^{2} u}{\partial y^{2}}
$$

One possible discretization of this equation uses a central difference of both forward- and backwards-difference discretizations of the first partial derivatives.

$$
\begin{gathered}
\frac{\partial^{2} u}{\partial x^{2}} \approx \frac{\frac{u_{i, j+1}-u_{i, j}}{h}-\frac{u_{i, j}-u_{i, j-1}}{h}}{h} \\
\frac{\partial^{2} u}{\partial y^{2}} \approx \frac{\frac{u_{i+1, j}-u_{i, j}}{h}-\frac{u_{i, j}-u_{i-1, j}}{h}}{h} \\
\frac{\partial^{2} u}{\partial x^{2}}+\frac{\partial^{2} u}{\partial y^{2}} \approx\left(\frac{1}{h^{2}}\right)\left(1 u_{i-1, j}+1 u_{i, j-1}-4 u_{i, j}+1 u_{i, j+1}+1 u_{i+1, j}\right)
\end{gathered}
$$

When applied to every point in the domain, the coefficient pattern $1,1,-4,1,1$ produces a five-banded square coefficent matrix $A$ in the equation

$$
A u=b
$$

where $u$ is the vector of unknowns, for which we must solve, and the right-hand side $b$ includes boundary information.

\section{Setup $R$ and $A$ Hierarchies}

The basic requirement of multigrid is, unsurprisingly, a multiplicy of grids, each discretizing the problem domain at a different resolution. In the simplest ("two-grid") scheme, there are two grid levels, $h$ and $H$, where grid $h$ has $N_{h}$ unknowns, grid $H$ has $N_{H}$ unknowns, $N_{h}>N_{H}$, and (for regular Cartesian grids) the values of $h$ and $H$ represent the fine and coarse grid spacings, respectively.

In geometric multigrid, the operator at the fine level $A_{h}$ is replaced by the operator at the coarse level $A_{H}$ by re-discretizing the underlying PDE. However, this method, while potentially faster, enforces a tighter coupling between the solver and the simulation problem at hand.

The alternative to geometric multigrid is algebraic multigrid, in which the coarse operator is derived not from the PDE but only from the fine operator. Ruge-Steuben coarsening bases this transformation on the pattern of coefficients in $A_{h}$, but our current implementation (see Implementation) instead uses a stencil-based average.

Before the cycling portion of the algorithm, a setup phase is executed in which we generate a hierarchy of restriction matrices and coefficient matrices. The restriction array at position $h$ in the hierarchy, where the number of unknowns is $N_{h}$, and where the number of unknowns for the next coarsest level is $N_{H}$, is $R_{h}^{H}$, or simply $R_{h}$. It functions as an intergrid transfer operator from grid $H$ to grid $h$, and has shape $\left(N_{H}, N_{h}\right)$. That is, it can reduce the size of a vector from $N_{h}$ to $N_{H}$ elements:

$$
u_{H}=R_{h} u_{h}
$$

These restriction matrices are used to produce a similar hierarchy of coefficient matrices, via the Galerkin coarse-grid approximation [Zeng].

$$
A_{H}=R_{h} A_{h} R_{h}^{T}
$$

This is significant because the multigrid algorithm thereby requires no knowledge of the underlying PDE to generate the coarsegrid operator. Instead, the coarse-grid operator is created solely through algebraic manipulation, giving rise to the term "algebraic multigrid".

It should be noted that the labels $h$ and $H$ are used because, in cartesian structured grids, the characteristic that distinguishes between grid levels is the spacing between points. It is geometrically intuitive to call the distance between points $h$ in the fine grid and $H$ in the coarse grid.

\section{Pre-Smoothing: $u_{a p x, h}$}

An iterative solver is used to produce an initial estimate of the solution. This solver can be a Jacobi, Gauss-Seidel, or conjugate gradient implementation, or any other solver that can use a number-of-iterations parameter to make a tradeoff between overall accuracy and speed.

These iterative solvers begin with some initial guess of the solution, which could either be the work of previous solvers or simply a zero-vector. Because the iterative solvers reduce the highfrequency components of the error in this guess more quickly than they reduce the low-frequency ones, they are often referred to as "smoothers" in the context of multigrid methods. The purpose of a multigrid scheme is to use these iterative smoothers only at high resolution to reduce the high-frequency error, relying on corrections at lower resolution to reduce the low-frequency components of the error. [Harimi] See Figure 5 c, and accompanying explanations in Test Definitions and Results. 
So,

$$
u_{\text {apx }, h}=\text { iterative_solve }\left(A_{h}, b_{h}, \text { iterations }\right)
$$

where iterations is a small integer, often simply 1 .

\section{Residual: $r_{h}$}

After the iterative solution, an error $r_{h}$ in the approximation $u_{a p x, h}$ can be defined as

$$
A_{h} u_{a p x, h}+r_{h}=b_{h}
$$

where $b_{h}$ is the given right-hand side.

\section{Coarse Right-hand-side: $b_{H}$}

let $r_{h}=A_{h} v_{h}$

$$
\begin{gathered}
A_{h} u_{a p x, h}+A_{h} v_{h}=b_{h} \\
A_{h}\left(u_{a p x, h}+v_{h}\right)=b_{h}
\end{gathered}
$$

So, $v_{h}$ functions as a correction vector for the iterative approximation. Equation 6 can be rearranged to produce another matrix equation in the same form as Equation 2:

$$
A_{h} v_{h}=b_{h}-A_{h} u_{a p x, h}
$$

Here, every element on the right-hand side is known, so it can be used to form a new right-hand side with which we can solve for the correction $v_{h}$. However, because this correction only serves the purpose of reducing the low-frequency components of the error, we can safely solve Equation 8 at a coarser resolution without losing information [Borzi]. So, we make use of our hierarchy of restriction and coefficient matrices to make Equation 8 an easier problem to solve (fewer unknowns):

$$
A_{H} v_{H}=R_{h}\left(b_{h}-A_{h} u_{a p x, h}\right)
$$

where $A_{H}$ and $R_{h}$ are taken from the hierarchy generated earlier.

\section{Coarse Solution}

The unknown vector and right-hand side of Equation 9 can now be replaced with new variables, revealing a new problem with only $N_{H}$ unknowns, down from the $N_{h}$ unknowns in Equation 8.

$$
A_{H} u_{H}=b_{H}
$$

Because this is simply another matrix equation similar in form to Equation 2, it can be solved either with a recursive call to the multigrid solver, or with a direct solver, such Numpy's np.linalg.solve or SciPy's scipy.base.np.linalg. solve.

\section{Interpolate Correction}

In order to correct the iterative approximation $u_{a p x}$, the solution from the coarse problem must be interpolated from $N_{H}$ unknowns up to $N_{h}$ unknowns. Because the restriction matrices are defined algebraically in Equation 3, it is possible to define an interpolation (or "prolongation") algebraically:

$$
v_{h}=R_{h}^{T} u_{H}
$$

This is used to prolongate the solution $u_{H}$ from the coarse level for use as a correction $v_{h}$ at the fine level. Note that, at the coarse level, the symbol $u$ is used, since this is a solution to the coarse problem, but, at the fine level, the symbol $v$ is used, since this is not the solution, but a correction to the iterative approximation.

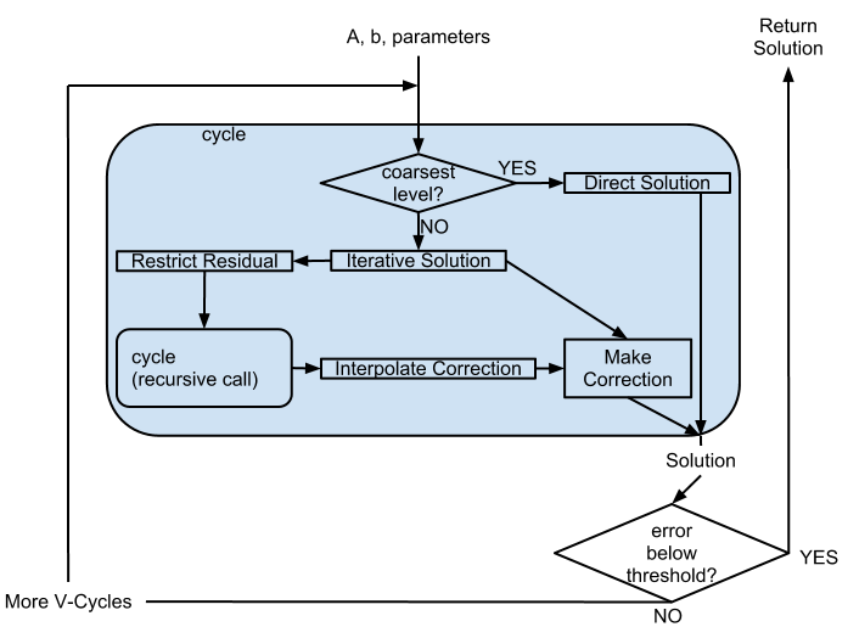

Fig. 1: Recursive multigrid cycle, with V-cycle iteration until convergence.

\section{Return Corrected Solution}

With the correction vector in hand, it is now possible to return a solution whose error has been reduced in both high- and lowfrequency components:

$$
u_{h}=u_{a p x}+v_{h}
$$

It is also possible to insert a second "post-smoothing" step between the interpolation and the return steps, similar to Equation 4.

As described in this section, this algorithm is a 2-grid V-cycle, because the high-resolution $\rightarrow$ low-resolution $\rightarrow$ high-resolution pattern can be visualized as a $\mathrm{V}$ shape. In our small sample problem, using more grid levels than two actually wasted enough time on grid setup to make the solver converge less quickly. However, repeated V-cycles were usually necessary for visually compelling convergence. That is, the solution from one V-cycle was used as the initial guess for the fine-grid pre-smoother of the next V-cycle. More complicated cycling patterns are also possible, such as W-cycles, or the full-multigrid ("FMG") pattern, which actually starts at the coarse level. However, these patterns are not yet addressed by OpenMG.

\section{Implementation}

The process shown in Figure 1 is a multigrid solver with nearly black-box applicability-the only problem-specific piece of information required (one of the "parameters" in the figure) is the shape of the domain, as a 3-tuple, and it is possible that future versions of restriction() will obviate this requirement. Note that, in code listings given below, import numpy as np is assumed.

\section{Setup $R$ and $A$ Hierarchies}

Any restriction can be described by a restriction matrix. Our current implementation, which is replacable in modular fashion, uses 2-point averages in one dimension, 4-point averages in two dimensions, and 8-point averages in three dimensions, as depicted in Figure 2. Alternate versions of these two functions have been developed that use sparse matrices, but the dense versions are shown here for simplicity. 


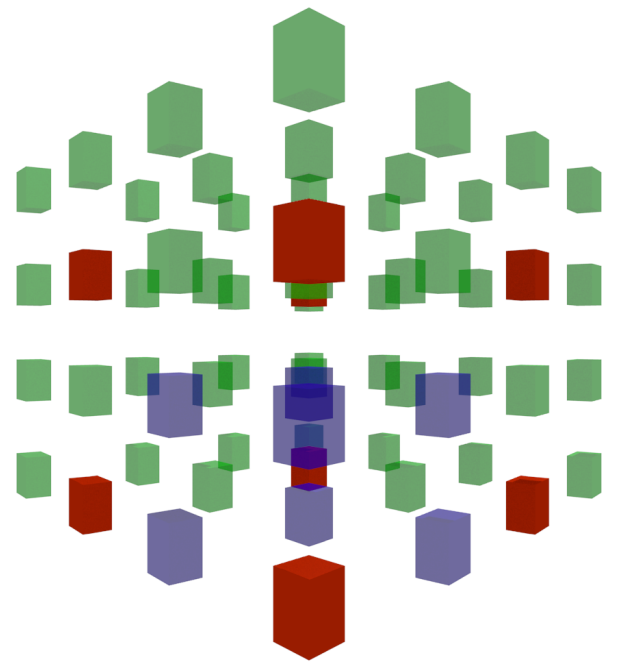

Fig. 2: Eight-point average restriction method. All points are included in the fine set, but red points included in both the fine set and the coarse set. Blue points are used in the calculation of eight-point average for the coarse point nearest to the camera in the bottom plane.

Other simplifications have also been made-for example, automatic V-cycling has been removed, although, in the actual code, this is contained with in the wrapper function openmg.mg_solve(). Forced line breaks have also reduced the readability of this sample code. We recommend downloading the most up-to-date OpenMG code from https://github.com/ tsbertalan/openmg for working examples.

The following code generates a particular restriction matrix, given a number of unknowns $\mathrm{N}$, and a problem domain shape tuple, shape. It fails (or works very inefficiently) for domains that have odd numbers of points along one or more dimensions. Operator-based coarsening would remove this restriction.

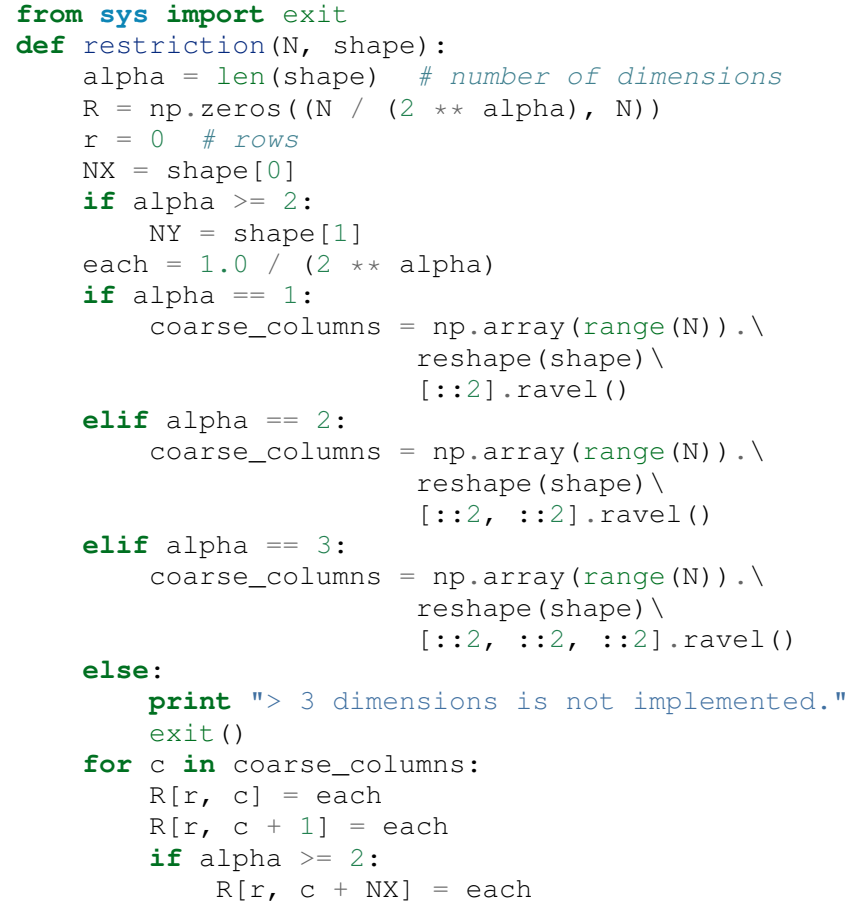

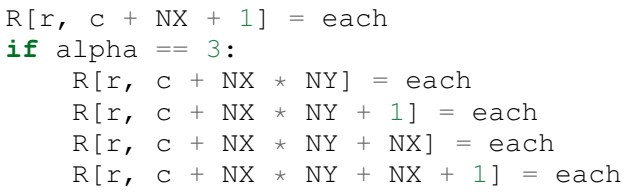

The function restriction() is called several times by the following code to generate the complete hierarchy of restriction matrices.

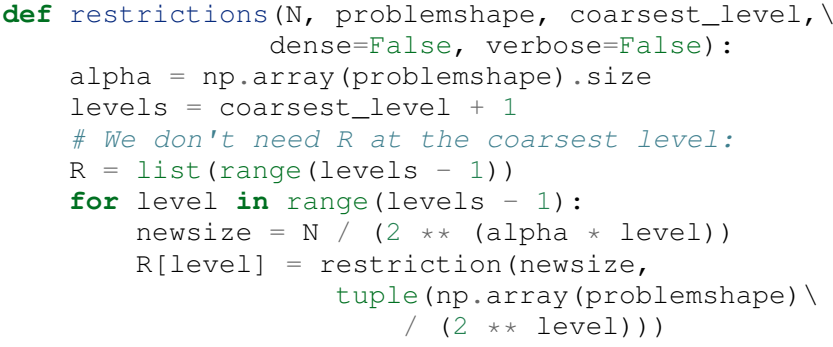

Using the hierarchy of restriction matrices produced by restrictions() and the user-supplied top-level coefficient matrix A_in, the following code generates a similar hierarchy of left-hand-side operators using the Galerkin coarse-grid approximation, $A_{H}=R A_{h} R^{T}$.

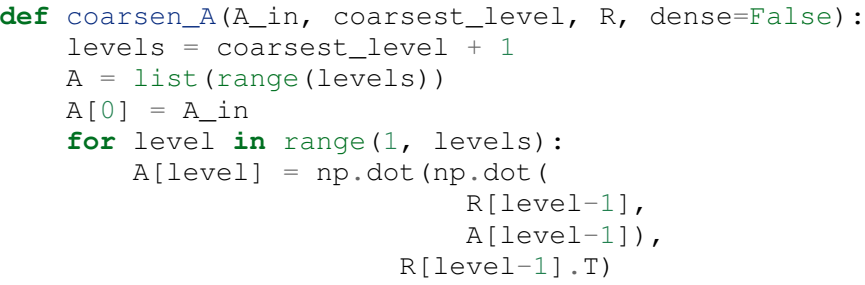

Both restrictions() and coarsen_A() return lists of arrays.

\section{Smoother}

Our iterative smoother is currently a simple implementation of Gauss-Seidel smoothing, but this portion of the code could be replaced with a Jacobi implementation to allow parallelization if larger domains prove to spend more execution time here.

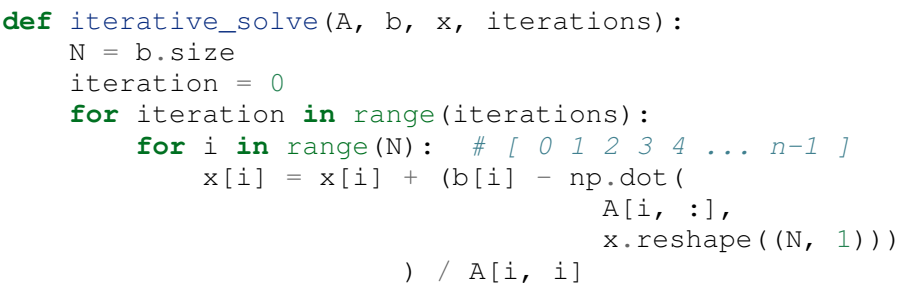

\section{Multigrid Cycle}

The following function uses all the preceeding functions to perform a multigrid cycle, which encompasses the Residual, Coarse Solution, Interpolate Correction, and Return Corrected Solution steps from the theoretical discussion above. It calls itself recursively until the specified number of gridlevels is reached. It can be called directly, or through a wrapper function with a more simplified prototype, mg_solve (A_in, b, parameters) (not shown here). 


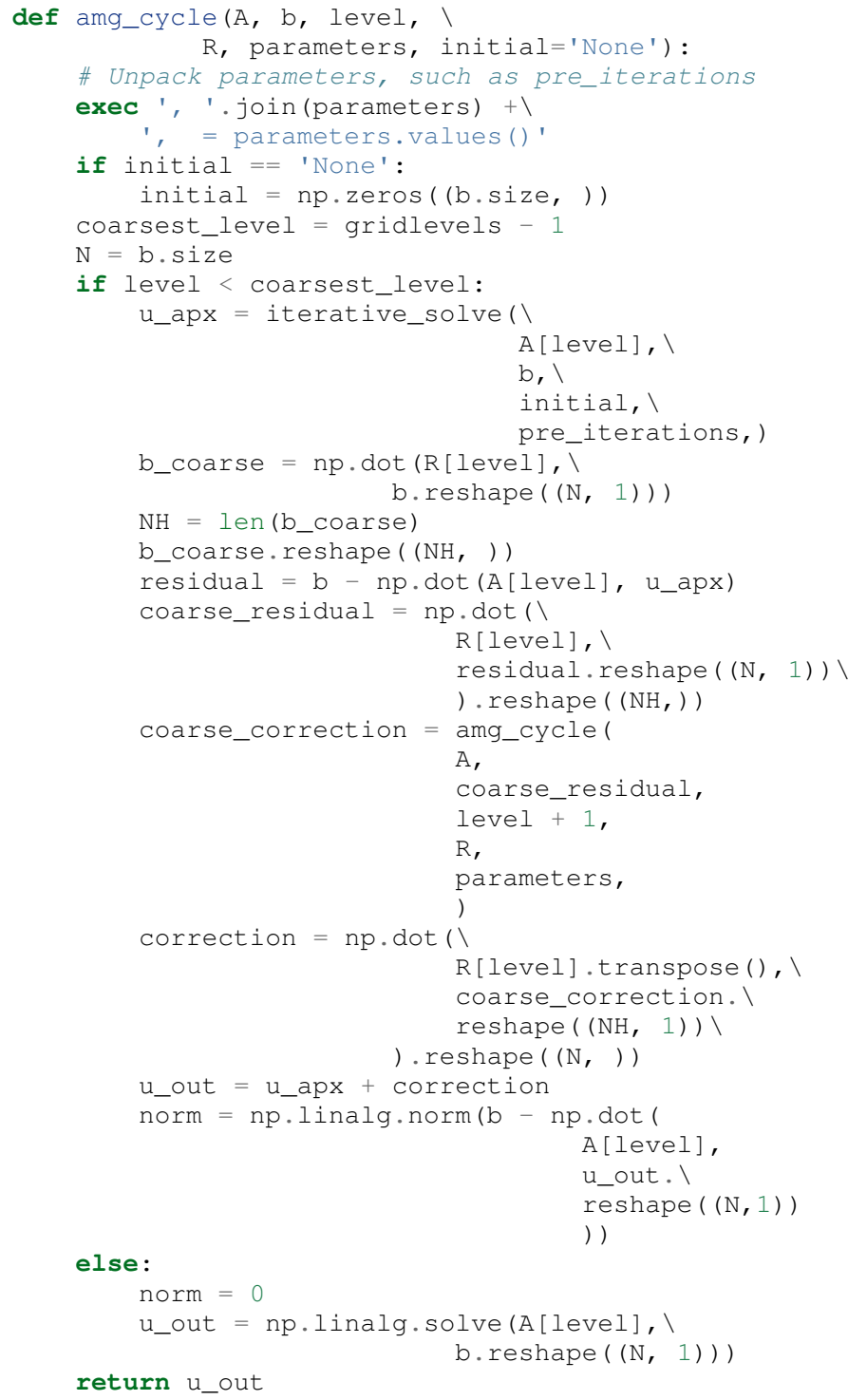

\section{Results}

\section{Sample Application}

In our test example we simulate the geologic sequestration of $\mathrm{CO}_{2}$. The governing pressure-saturation equation is

$$
v=-\mathbf{K}\left(\lambda_{w}+\lambda_{\mathrm{CO}_{2}}\right) \nabla p+\mathbf{K}\left(\lambda_{w} \rho_{w}+\lambda_{\mathrm{CO}_{2}} \rho_{\mathrm{CO}_{2}}\right) G
$$

and the saturation equation is

$$
\phi \frac{\partial s_{w}}{\partial t}+\nabla\left(f_{w}\left(s_{w}\right)\left[v+d\left(s_{w}, \nabla s_{w}\right)+g\left(s_{w}\right)\right]\right)=\frac{q_{w}}{\rho_{w}}
$$

where $v$ is a velocity vector, the gravitational pull-down force $G$ is $-g \nabla z$, subscript $w$ represents water-saturated porous medium, $g$ represents gravitational acceleration, $\mathbf{K}$ represents the permeability tensor, $p$ represents fluid pressure, $q$ models sources and sinks, (outflow or inflow), $S$ represents saturation, $z$ represents the vertical direction, $\rho$ represents water density, $\phi$ represents porosity, and $\lambda$ represents mobility (ratio of permeability to viscosity).

Equation 14, the saturation equation, is generally parabolic. However, the terms for the viscous force $f(s) v$ and the gravity force $f(s) g(s)$ usually dominate the capillary force $f(s) d(s, \nabla s)$. Therefore the equation will have a strong hyperbolic nature and

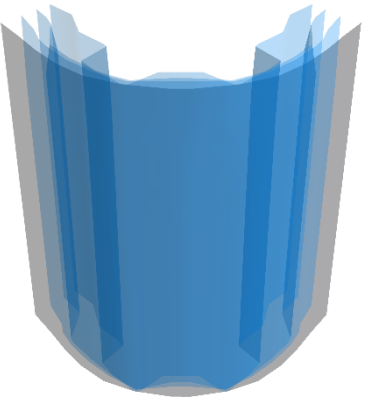

Fig. 3: Pressure isosurfaces of several solutions to a $3 D$ porous media problem with $12^{3}=1728$ unknowns. The grey outer surface is a direct solution, while the blue inner surfaces are the result of different numbers of multigrid V-cycles-with more V-cycles, the multigrid solution approaches the true solution. Plotted with MayaVi's mlab. contour 3 d.

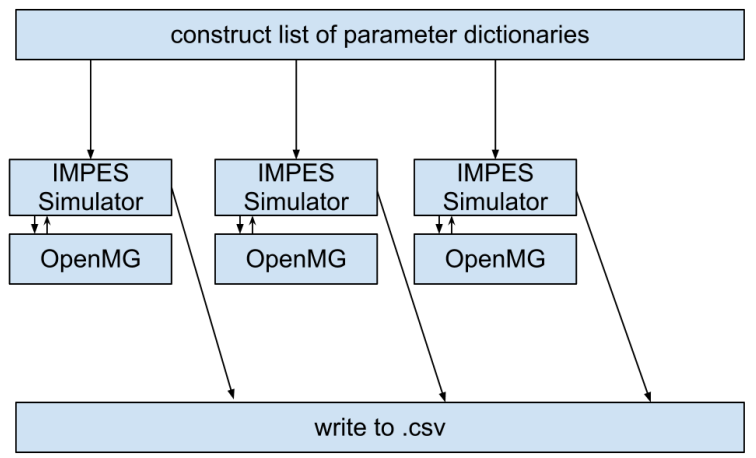

Fig. 4: Parallel testing apparatus. The IMPES (implicit pressure, explicit saturation) simulation script calls the OpenMG script when solving its pressure equation, and then reports a dictionary of dependent variables of interest to be written to a comma-separated-value file.

can be solved by many schemes [Aarnes]. On the other hand, Equation 13, the pressure equation, is of elliptic form. After discretization, this equation will reduce to $A u=b$ and a multigrid scheme can be used for efficient computation especially if the problem size is big (for instance, millions of cells [Carlson]).

The unknown quantity, which the solver algorithm must find, is the fluid pressure $p$. In Figure 3, we show 2033 psi isosurfaces of this solution (pressure across the entire domain varies by only about 5 psi). The actual solution (via np.linalg. solve) is rendered in grey, and the three blue surfaces (from narrowest to widest) are the result of applying one, two, and three two-grid cycles, respectively.

As shown, this two-grid solver is converging on the true solution in the vicinity of this isosurface. The multigrid isosurface and the direct solution isosurface become indistinguishable within about ten V-cycles.

\section{Discussion}

\section{Testing Setup}

In a wrapper script depicted in Figure 4, we used the Python 2.6 module multiprocessing.Pool to accelerate the execution of test sets. A dictionary of parameters is constructed for each 
distinct possible parameter combination where several parameters of interest are being varied. A process in the pool is then assigned to test each parameter combination. Each pool process then returns a dictionary of dependent variables of interest. Our tests are run on a dual-socket Intel Xeon E5645 $(2.40 \mathrm{GHz})$ machine with 32 GB of memory. However, care still must be taken to ensure that the number of processes in the pool is not so high that individual processes run out of memory.

\section{Test Definitions and Results}

In Figure 5 a, we show the results of a V-cycle convergence test with our OpenMG solver. Here, we specify the number of repeated 2-grid cycles as an independent variable, and monitor the residual norm as the dependent variable. There were $8^{3}=512$ unknowns, one pre-smoothing iteration, and zero post-smoothing iterations. OpenMG was able to reduce the error at a steady logarithmic rate. The norm used everywhere was the 2-norm.

This contrasts with Figure 5 b, where we show the convergence behavior of the ordinary Gauss-Seidel on its own. Similarly to the method used for Fig. 5 a, we used the number of iterations as the independent variable, and examined the residual norm as the dependent variable. There were $12^{3}=1723$ unknowns, and the test took 43 hours to complete 200,000 iterations. However (for this sample problem), the Gauss-Seidel solver quickly exhausts the high-frequency portions of the solution error, and begins slower work on the low-frequency components.

This frequency-domain effect can be seen more clearly in Figure $5 \mathrm{c}$, where we show the Fourier transform of the error $\left(u-u_{a p x}\right)$ after different numbers of Gauss-Seidel iterations. A Hann-window smoother with a window width of 28 was applied after the Fourier transform to better distinguish the several curves. For this test, we used a 1D Poisson coefficent matrix and an expected solution vector generated using $\mathrm{np}$.random.random $((\mathrm{N})$,$) . reshape ((\mathrm{N}, 1))$, where $\mathrm{N}$ was 18,000 unknowns. Because of this method of noise generation (a continuous uniform distribution, or equal probability of all permitted magnitudes at all points in the domain), the pre-generated solution sampled all frequencies unequally, unlike true white noise. This accounts for the initial bell-shaped error in the frequency domain. However, the unequal rate of errorreduction for different frequencies that was observed as iterations were completed is to be expected of iterative solvers, hence their description as "smoother" in the context of multigrid methods. This recalls the argument from a frequency-domain perspective for a multigrid solver [Brandt2].

In Figure $5 \mathrm{~d}$, we examine the effect of this Gauss-Seidel presmoother by increasing the number of pre-smoothing iterations from our default value of only one. Dependent variables include the number of $\mathrm{V}$-cycles required to obtain a residual norm of 0.00021 , and the time taken by the whole OpenMG solver to arrive at that precision. There were $8^{3}=512$ unknowns and two grid levels, and all restriction and coefficient matrices used were stored in dense format. As expected, increasing the number of pre-smoothing iterations does decrease the number of required Vcycles for convergence, but this does not generally improve the solution time, except in the transition from $3 \mathrm{~V}$-cycles to $2 \mathrm{~V}$ cycles. However, this trend is useful to validate that the smoother is behaving as expected, and might be useful if, in the future, some coarsening method is employed that makes V-cycling more expensive. a. Precision achieved vs. number of v-cycles.

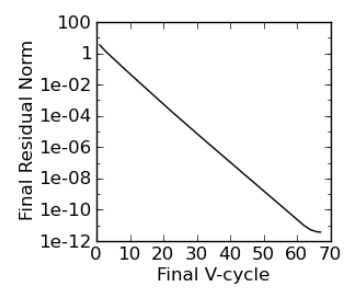

b. Gauss-Seidel solution rate.

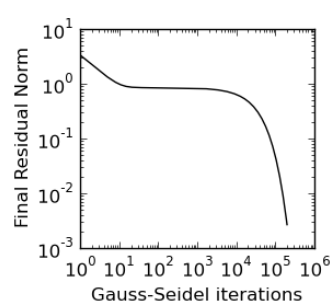

c. Fourier transform of error for several numbers of GS iterations.

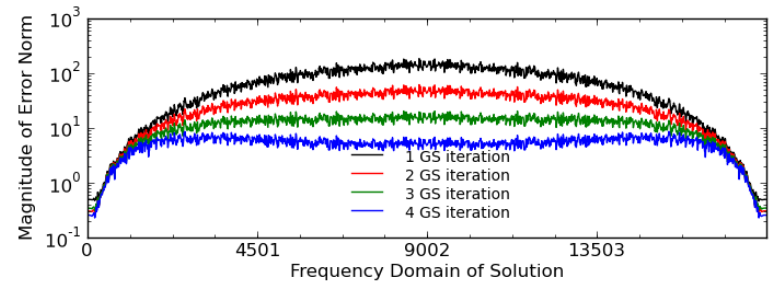

d. Effect of pre-smoothing on required $v$-cycles and solution time.

e. Time taken for several solvers.
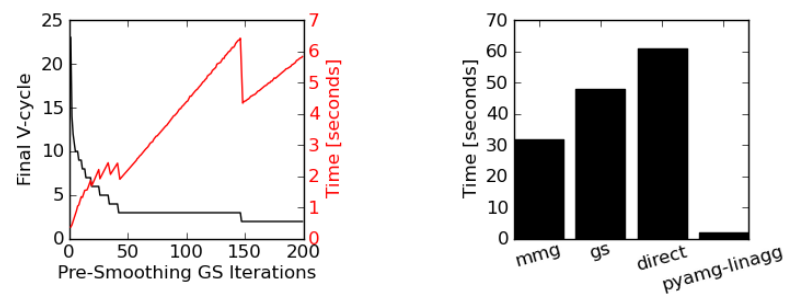

Fig. 5: Results from explanatory tests. Tests described and interpreted in Test Definitions and Results.

The Gauss-Seidel (GS) solver's very slow convergence in lowfrequency error accounts for the difference in time between it and the OpenMG multigrid (mmg) solver, as shown in Figure 5 e. Here, we compare the running times of several solvers, including PyAMG's smoothed aggregation solver, our own pure-python Gauss-Seidel iterative solver, and the direct solver $n p$.linalg.solve. There were $20^{3}=8000$ unknowns, and dense $R$ and $A$ matrices were used for OpenMG. In order to keep the GS bar similar in scale to the other bars in the chart, a relatively high residual norm tolerance of 0.73 was used for both the GS and $\mathrm{mmg}$ solvers. However, this tolerance parameter was not an option for the direct solver or PyAMG, both of which achieved very good precision without prompting. The PyAMG solver (pyamg-linagg) used linear aggregation coarsening, and so is not really comparable to our multigrid implementation in this example, but it is included in this plot to demonstrate the speed that can be achieved using optimized multigrid methods with efficient coarsening algorithms. Our own coarsener uses the simple geometric scheme shown in Figure 2, not the more efficient, general, and geometry-agnostic Ruge-Steuben method usually used in algebraic multigrid solvers.

\section{Conclusion and Future Work}

OpenMG is an environment for testing new implementations of algebraic multigrid components. While optimized implementations such as PyAMG are more suitable for use as production solvers, OpenMG serves as an easy-to-read and easy-to-modify implementation to foster understanding of multigrid methods. For example, future module improvements could include a parallel 
Jacobi iterative solver, a method of generating restriction matrices that is tolerant of a wider range of problem sizes, or operator-based Ruge-Steuben coarsening in addition to the option of stencil-based coarsening. In order to find computational bottlenecks, it might be useful also to add a per-step time profiler.

As open-source software, the code for this project has been posted online under the New BSD license at https://github.com/ tsbertalan/openmg. We invite the reader to download the code from this address to explore its unit tests and possible modifications, and to contribute new modules.

\section{REFERENCES}

[AGMG] Y Notay, $A G M G$, 2012. [Online]. Available: http://homepages. ulb.ac.be/ ynotay/AGMG.

[Aarnes] J E Aarnes, T Gimes, and $\mathrm{K}$ Lie. An Introduction to the Numerics of Flow in Porous Media using Matlab, Geometric Modeling, Numerical Simulation and Optimization. 2007, part II, 265-306.

[Bakshi] B R Bakshi and G Stephanopoulos, Wave-net: a multiresolution, hierarchical neural network with localized learning, AIChE Journal, vol. 39, no. 1, pp. 57-81, Jan. 1993.

[Bell] N Bell, L Olson, and J Schroder, PyAMG: Algebraic Multigrid Solvers in Python, 2011.

[Borzi] A Borzi, Introduction to multigrid methods. [Online]. Available: http://www.uni-graz.at/imawww/borzi/mgintro.pdf. [Accessed: 03-Jul-2012].

[Boschitsch] A H Boschitsch and M O Fenley, A Fast and Robust PoissonBoltzmann Solver Based on Adaptive Cartesian Grids., Journal of chemical theory and computation, vol. 7, no. 5, pp. 15241540, May 2011.

[Brandt1] A Brandt, Rigorous Quantitative Analysis of Multigrid I. Constant Coefficients Two-Level Cycle with L2 Norm, SIAM Journal on Applied Mathematics, vol. 31, no. 6, pp. 1695-1730, 1994.

[Brandt2] A Brandt, Multi-Level Adaptive Solutions to Boundary-Value Problems, Mathematics of Computation, vol. 31, no. 138, pp. 333-390, 1977.

[Brandt3] A Brandt, Multilevel computations of integral transforms and particle interactions with oscillatory kernels, Computer Physics Communications, vol. 65, no. 1-3, pp. 24-38, Apr. 1991.

[Brandt4] A Brandt, $A M G$ and Multigrid Time-Dependence, Multigrid Methods: Lecture Notes In Mathematics, pp. 298-309, 1987.

[Carlson] E S Carlson, A W Islam, F Dumkwu, and T S Bertalan. nSpyres, An OpenSource, Python Based Framework for Simulation of Flow through Porous Media, 4th International Conference on Porous Media and Annual Meeting of the International Society for Porous Media, Purdue University, May 14-16, 2012.

[Denev] J A Denev, F Durst, and B Mohr, Room Ventilation and Its Influence on the Performance of Fume Cupboards: A Parametric Numerical Study, Industrial \& Engineering Chemistry Research, vol. 36, no. 2, pp. 458-466, Feb. 1997

[Douglas1] C C Douglas, L I Deng, G Haase, M Liebmann, and R Mckenzie, Amglab: a community problem solving environment for algebraic multigrid methods. [Online]. Available: http://www.mgnet.org/mgnet/Codes/amglab.

[Douglas2] C C Douglas, J Hu, M Iskandarani, M Kowarschik, U Rüde, and C Weiss, Maximizing Cache Memory Usage for Multigrid Algorithms for Applications of Fluid Flow in Porous Media, vol. 552. Berlin, Heidelberg: Springer Berlin Heidelberg, 2000.

[Dzwinel] W Dzwinel, D. A. Yuen, and K. Boryczko, Bridging diverse physical scales with the discrete-particle paradigm in modeling colloidal dynamics with mesoscopic features, Chemical Engineering Science, vol. 61, no. 7, pp. 2169-2185, Apr. 2006.

[Fedorenko1] R P Fedorenko, The Speed of Convergence of One Iterative Process, Zhurnal Vychislitel'noi Matematiki i Matematicheskoi Fiziki, 1964.

[Fedorenko2] R P Fedorenko, A relaxation method for solving elliptic difference equations, Zhurnal Vychislitel'noi Matematiki i Matematicheskoi Fiziki, pp. 922-927, 1961.

[Hackbusch] W Hackbusch, Multi-Grid Methods and Applications. Springer, 1985 , p. 377.

[Harimi] I Harimi and M Saghafian, Evaluation of the Capability of the Multigrid Method in Speeding Up the Convergence of Iterative Methods, ISRN Computational Mathematics, vol. 2012, pp. 1-5, 2012.
[Heckbert] P Heckbert, Survey of Multigrid Applications, 1998. [Online]. Available: http://www.cs.cmu.edu/ ph/859E/www/notes/ multigrid.pdf. [Accessed: 13-Jun-2012].

[Kameswaran] S Kameswaran, L T Biegler, and G H Staus, Dynamic optimization for the core-flooding problem in reservoir engineering, Computers \& Chemical Engineering, vol. 29, no. 8, pp. 1787 1800, Jul. 2005.

[Nicolaides] R A Nicolaides, On Some Theoretical and Practical Aspects of Multigrid Methods, Mathematics of Computation, 1979. [Online]. Available: http://www.jstor.org/stable/10.2307/2006069. [Accessed: 07-Jul-2012].

[Notay] Y Notay, An aggregation-based algebraic multigrid method, Electronic Transactions on Numerical Analysis, vol. 37, pp. 123-146, 2010

[Trottenberg] U Trottenberg, C W Oosterlee, and A Schüller, Multigrid, Academic Press, 2001, p. 631

[Wesseling] P Wesseling, An introduction to multigrid methods. 1992, Willey, New York, 1991.

[Zapata] G Zapata-Torres et al., Influence of protonation on substrate and inhibitor interactions at the active site of human monoamine oxidase-a., Journal of chemical information and modeling, vol. 52, no. 5, pp. 1213-21, May 2012.

[Zeng] S Zeng and P Wesseling, Galerkin Coarse Grid Approximation for the Incompressible Navier-Stokes Equations in General Coordinates, Thesis, 2010. 